\title{
Comparison of Dose Distributions When Using Carbon Ion Radiotherapy Versus Intensity-modulated Radiotherapy for Hepatocellular Carcinoma With Macroscopic Vascular Invasion: A Retrospective Analysis
}

\author{
SHINTARO SHIBA ${ }^{1,2}$, KEI SHIBUYA ${ }^{1}$, MOTOHIRO KAWASHIMA $^{2}$, NAOKO OKANO $^{1}$, TAKUYA KAMINUMA $^{1}$, \\ MASAHIKO OKAMOTO ${ }^{1}$, YOSHIKI KUBOTA ${ }^{2}$, TAKASHI NAKANO ${ }^{1}$ and TATSUYA OHNO ${ }^{2}$ \\ ${ }^{1}$ Department of Radiation Oncology, Gunma University Graduate School of Medicine, Maebashi, Japan; \\ ${ }^{2}$ Gunma University Heavy Ion Medical Center, Maebashi, Japan
}

\begin{abstract}
Background/Aim: This study compared the dose distributions of carbon ion radiotherapy $(C$-ion $R T)$ and intensity-modulated radiotherapy (IMRT) in patients with locally advanced hepatocellular carcinoma (LAHCC). Patients and Methods: A retrospective analysis was conducted in 10 consecutive patients with LAHCC who had undergone C-ion $R T$. The dose-volume histogram parameters of clinical plans using C-ion RT at 60 Gy and simulated plans using IMRT at 60 Gy and 50 Gy were compared. We measured the percentage of the normal liver volume that received at least $5 \mathrm{~Gy}\left(V_{5}\right), 10$ $G y\left(V_{10}\right), 20 G y\left(V_{20}\right), 30 G y\left(V_{30}\right), 40 G y\left(V_{40}\right)$, and $50 G y$ $\left(V_{50}\right)$. Results: The $V_{5}, V_{10}, V_{20}$, and the mean liver dose were significantly lower in patients who received $60 \mathrm{~Gy}$ of $\mathrm{C}$-ion $R T$ than in those who received 50 or 60 Gy of IMRT. Conclusion: $C$-ion RT exhibits a better liver dose distribution than IMRT in patients with LAHCC.
\end{abstract}

Hepatocellular carcinoma (HCC) involving a major branch of the portal or hepatic vein, including portal vein tumor thrombosis (PVTT) or inferior vena cava tumor thrombosis (IVCTT), are locally advanced-stage tumors that are difficult to treat despite the availability of multidisciplinary therapies. In a randomized controlled trial, Yoon et al. found that the clinical outcomes of patients with locally advanced HCC

This article is freely accessible online.

Correspondence to: Shintaro Shiba, Department of Radiation Oncology, Gunma University Graduate School of Medicine, 3-3922, Syowa-machi, Maebashi, Gunma 371-8511, Japan. Tel: +81 272208383, Fax: +81 272208397, e-mail: shiba4885@yahoo.co.jp

Key Words: Carbon ion radiotherapy, intensity-modulated radiotherapy, hepatocellular carcinoma, vascular invasion.
(LAHCC) who underwent transarterial chemoembolization (TACE) combined with X-ray radiotherapy (RT) were more favorable than those of patients only treated with sorafenib (1), and that TACE combined with X-ray RT improved survival compared to sorafenib alone. Accordingly, TACE combined with X-ray RT could potentially be a standard treatment for patients with LAHCC.

Conventional X-ray RT is of limited use owing to the poor radiation tolerance of the liver; therefore, a curative dose to the tumor is usually not feasible. More recently, stereotactic body RT (SBRT) and intensity-modulated RT (IMRT), which can deliver high radiation doses to the target with much better precision, have become widely used to treat patients with HCC (2-4). Particle therapy has since improved radiation dose distributions further still $(5,6)$. In particular, carbon ion RT (C-ion RT) has produced favorable clinical outcomes for patients with HCC (7-11), as this modality reduces the dose to the liver while ensuring good target coverage $(12,13)$ owing to its physical characteristics that include distal tail-off because of a high and narrow Bragg Peak as well as a sharp lateral penumbra (14). Abe et al.'s dosimetric comparison between C-ion RT and SBRT for HCC treatment found that the former has better sparing of the liver (5). However, their study investigated patients with HCC that exhibited no macroscopic vascular invasion; there have been no studies comparing the dose distributions of C-ion RT and IMRT in patients with LAHCC. Nevertheless, it is important to compare the dose distributions of C-ion RT and IMRT for patients with LAHCC (which have large and/or irregular shapes), as the dose to the liver may increase for the patients with LAHCC than the patients with HCC who have no macroscopic vascular invasion. As such, we conducted a simulation analysis to compare the dose distributions of C-ion RT and IMRT in patients with LAHCC. 


\section{Patients and Methods}

Patients. Data from patients with HCC who had undergone C-ion RT at Gunma University Heavy Ion Medical Center between June 2010 and November 2018 were retrospectively collected. A total of 210 HCC patients received C-ion RT during the study period; 10 patients with LAHCC (i.e., HCC involving a major branch of the portal or hepatic vein, including the PVTT or IVCTT) were investigated. The diagnoses of all patients were confirmed by the presence of typical hallmarks of HCC using four-phase multidetector-row computed tomography (CT) or dynamic contrastenhanced magnetic resonance imaging. This study complied with the standards set forth in the Declaration of Helsinki and current ethical guidelines, and was reviewed and approved by our Institutional Review Board (approval number1608).

Target delineation. Treatment-planning CT with respiratory-gated $\mathrm{CT}$, as well as four-dimensional CT images were acquired. Treatment-planning and contrast-enhanced CT images were merged to precisely delineate the gross tumor volume (GTV). The clinical target volume (CTV) was defined as the GTV plus at least a $5 \mathrm{~mm}$ margin in all directions to encompass any microscopic disease progression. The internal target volume (ITV) was defined as the sum of CTV from the four-dimensional CT images. The planning target volume (PTV) was defined as the sum of the ITV and an additional margin for patient setup discrepancies (8).

$C$-ion RT. At our facility, the C-ion RT dose distribution was calculated using the $\mathrm{XiO}-\mathrm{N}$, software, which is based on the $\mathrm{XiO}$ treatment planning system (Elekta, Stockholm, Sweden) and incorporates an ion beam RT dose engine (K2 dose) developed by the National Institute of Radiological Sciences (15) as well as a source management tool developed by Mitsubishi Electric (Tokyo, Japan). C-ion RT doses were expressed in Gy [relative biological effectiveness (RBE)], which was defined as the physical dose multiplied by the RBE of C-ions (16). The heavy ion accelerator at our facility generated $\mathrm{C}$-ion beams; the passive scattering technique was applied for the treatment of HCC. The beam energy selections were $290 \mathrm{MeV} / \mathrm{u}, 380 \mathrm{MeV} / \mathrm{u}$, or $400 \mathrm{MeV} / \mathrm{u}$ depending on the depth of the tumor. Prescribed doses were $60 \mathrm{~Gy}(\mathrm{RBE})$, with the aim of covering the PTV with at least $95 \%$ of this dose. C-ion RT plans used two or three coplanar ports to avoid the gastrointestinal (GI) tract. The maximum dose to $1 \mathrm{ml}\left(\mathrm{D}_{1 \mathrm{cc}}\right)$ was $<40 \mathrm{~Gy}$ (RBE) administered to the GI tract (8). Figure 1 shows the typical dose distribution of $\mathrm{C}$ ion RT; all treatment plans were made by medical physicists.

$I M R T$. IMRT treatment plans were calculated using the X-ray RT treatment-planning system (Eclipse, Varian Medical Systems, Inc., CA, USA). All IMRT treatment plans were simulated using the treatment-planning CT images of patients who received C-ion RT; moreover, all the contours were the same as those used for C-ion RT. The prescribed doses were 60 and $50 \mathrm{~Gy}$; the aim was to cover the PTV with at least $95 \%$ of the prescribed dose. IMRT plans used five or six coplanar $6 \mathrm{MV} \mathrm{X}$-rays with gantry angles to avoid the GI tract and spinal cord. The dose constraints were $\mathrm{D}_{1 \mathrm{cc}}<40 \mathrm{~Gy}$ administered to the GI tract. The weights of each field were also arranged to cover the PTV with at least $95 \%$ of the prescribed dose while minimizing the dose to the liver and GI tract. Figure 2 shows the typical IMRT dose distribution in the same patient as that shown in Figure 1; again, all IMRT treatment plans were made by medical physicists.
Dose-volume histogram (DVH) parameters and statistical analyses. We assessed the percentage of the minimum dose that covered $95 \%$ of the target volume $\left(\mathrm{D}_{95}\right)$ based on the DVH for the PTV. To compare normal tissue sparing achieved with $\mathrm{C}$-ion RT versus IMRT, we assessed the mean liver dose (MLD) as well as the percentage of the normal liver volume that received at least $5,10,20,30,40$, and $50 \mathrm{~Gy}$ using both C-ion RT (RBE) and IMRT (the $\mathrm{V}_{5}, \mathrm{~V}_{10}, \mathrm{~V}_{20}, \mathrm{~V}_{30}, \mathrm{~V}_{40}$, and $\mathrm{V}_{50}$, respectively) for the normal liver (i.e., total liver volume minus GTV). Additionally, we defined patients at high risk for developing radiation-induced liver disease (RILD) as those with MLD $>23$ Gy and $V_{30}$ values $>28 \%$ (17).

The DVH parameters when using both modalities were analyzed. Normal distributions were compared using $t$-tests (the $\mathrm{V}_{5}, \mathrm{~V}_{10}, \mathrm{~V}_{20}$, $\mathrm{V}_{30}, \mathrm{~V}_{40}, \mathrm{~V}_{50}$, and MLD), and non-normal distributions were compared using Wilcoxon's test (PTV $\mathrm{D}_{95}$ ), after testing for normality with the Shapiro-Wilk test. Statistical significance was defined as a $p$-value less than 0.05. All statistical analyses were performed using the SPSS 22.2.0 software (IBM Corp, Armonk, NY, USA).

\section{Results}

Patient characteristics. The median tumor size was $53 \mathrm{~mm}$ (range=27-119 mm). The median PTV and liver volume were $363.2 \mathrm{~cm}^{3}\left(\right.$ range $\left.=77.1-1331.2 \mathrm{~cm}^{3}\right)$ and $1113.3 \mathrm{~cm}^{3}$ (range $=782.6-1854.6 \mathrm{~cm}^{3}$ ), respectively.

DVH parameters. Table I shows the DVH parameters used in the clinical plans of C-ion RT [60 Gy (RBE)] versus those used in the simulated IMRT using $60 \mathrm{~Gy}$, while Table II shows the same $\mathrm{C}$-ion RT plan compared to the simulated IMRT using $50 \mathrm{~Gy}$.

There was no significant difference in the PTV $\mathrm{D}_{95}$ between $60 \mathrm{~Gy}$ (RBE) with C-ion RT and $60 \mathrm{~Gy}$ with IMRT $(p=0.24)$. In contrast, the difference in the PTV $\mathrm{D}_{95}$ between 60 Gy (RBE) with C-ion RT and 50 Gy with IMRT was significant $(p<0.01)$.

There were also significant differences in the liver $\mathrm{V}_{5}$, $\mathrm{V}_{10}$, and $\mathrm{V}_{20}$ between the $60 \mathrm{~Gy}$ (RBE) C-ion RT plan and each of the $60 \mathrm{~Gy}$ and 50 Gy IMRT plans $(p<0.05$ for both comparisons). In contrast, there were no significant differences in the $\mathrm{V}_{30}, \mathrm{~V}_{40}$, and $\mathrm{V}_{50}$, values, indicating high-dose areas. Thus, the median MLD was also significantly different between C-ion RT at 60 Gy (RBE) and IMRT at $60 \mathrm{~Gy}$.

The numbers of patients who exceeded an MLD of 23 Gy and $\mathrm{V}_{30}$ of $28 \%$ in the C-ion RT 60 Gy (RBE) group were 1 and 4, respectively, those in the IMRT 60 Gy group were 7 and 8 , respectively, and those in the IMRT $50 \mathrm{~Gy}$ group were 6 and 7 , respectively. Therefore, the numbers of patients at high risk of developing RILD in the three groups were 1,7 , and 6 , respectively. Scatterplots of the MLD and tumor sizes when comparing these modalities are shown in Figure 3. 


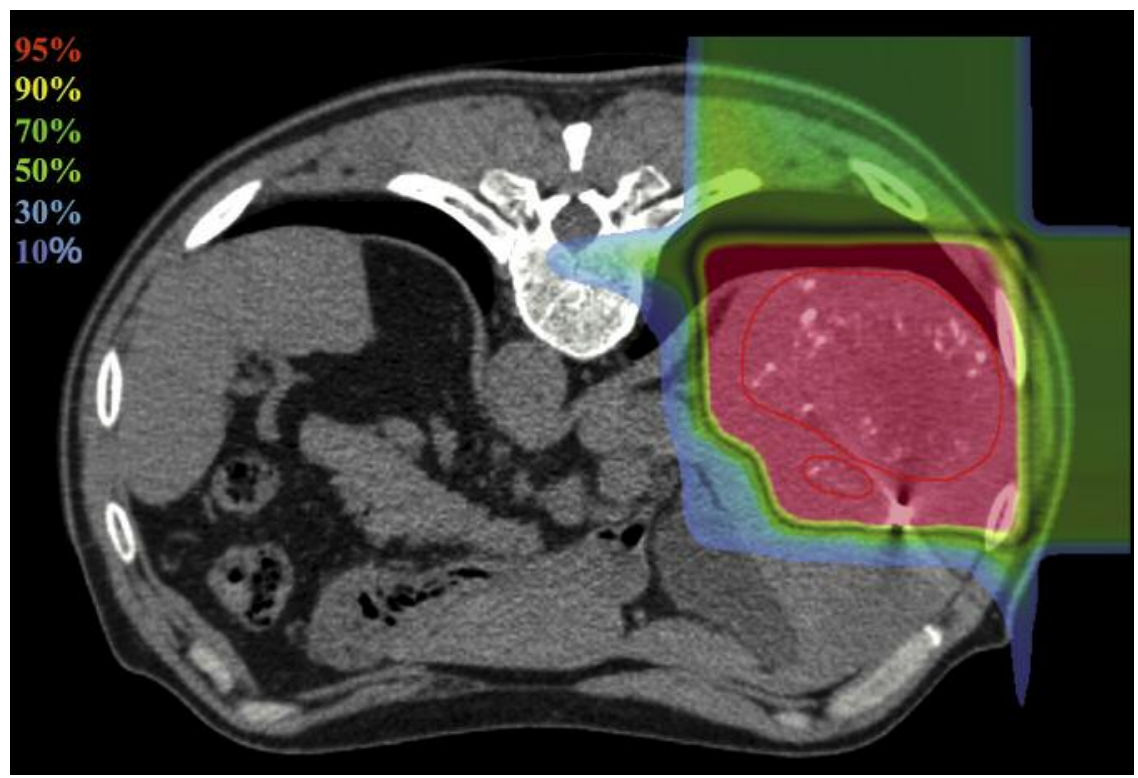

Figure 1. Axial computed tomography images of a locally advanced hepatocellular carcinoma. The dose distribution is shown, when treated with 60 Gy [relative biologic effectiveness (RBE)] of carbon-ion radiotherapy. The area within the red outline is the gross target volume. Highlighted are the $95 \%$ (red), $90 \%$ (yellow), 70\% (light green), 50\% (green), 30\% (light blue), 10\% (blue) isodose curves [100\% was 60 Gy (RBE)].

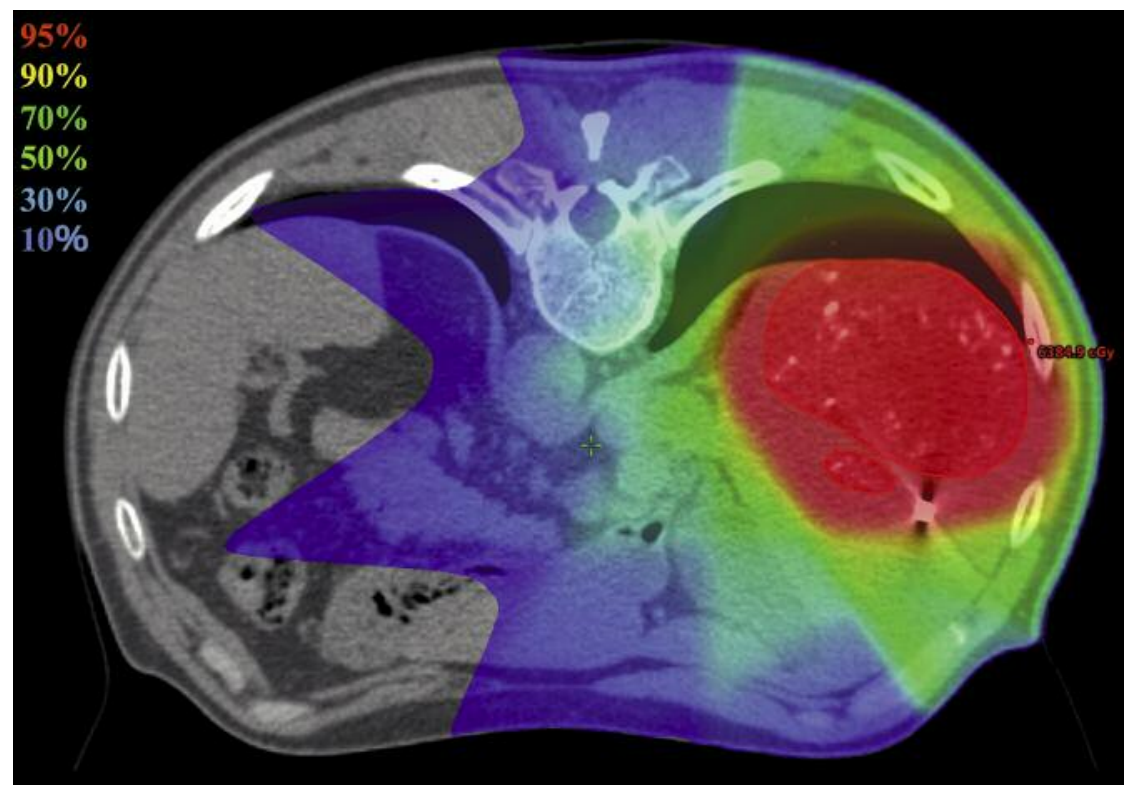

Figure 2. Axial computed tomography images of a locally advanced hepatocellular carcinoma. The dose distribution is shown, when treated with 60 Gy with IMRT. The area within the red outline is the gross target volume. Highlighted are the 95\% (red), 90\% (yellow), 70\% (light green), 50\% (green), 30\% (light blue), 10\% (blue) isodose curves (100\% was 60 Gy). The tumor is from the same patient as that shown in Figure 1.

\section{Discussion}

Our study demonstrated that C-ion RT has a more favorable dose distribution than IMRT when treating LAHCC, and also produces a lower dose to the liver. Additionally, there was a significant difference in PTV dose coverage between $\mathrm{C}$-ion RT at 60 Gy (REB) and IMRT at 50 Gy.

A number of studies have investigated the relationship between liver DVH parameters and RILD given that this 
Table I. DVH parameters of C-ion RT [60 Gy (RBE)] and simulated IMRT (60 Gy).

\begin{tabular}{|c|c|c|c|}
\hline & Median (range) for C-ion RT at $60 \mathrm{~Gy}$ (RBE) & Median (range) for IMRT at $60 \mathrm{~Gy}$ & $p$-Value \\
\hline PTV D 95 & $58.5 \mathrm{~Gy}(\mathrm{RBE})[\mathrm{range}=49.1-60.0 \mathrm{~Gy}(\mathrm{RBE})]$ & 58.9 Gy $($ range $=55.6-60.0 \mathrm{~Gy})$ & $p=0.29$ \\
\hline \multicolumn{4}{|c|}{ (16) } \\
\hline $\mathrm{V}_{5}$ & $41.0 \%($ range $=12.1-76.2 \%)$ & $85.3 \%($ range $=52.5-99.8 \%)$ & $p<0.01$ \\
\hline $\mathrm{V}_{10}$ & $34.5 \%($ range $=10.6-71.2 \%)$ & $72.6 \%($ range $=32.9-96.7 \%)$ & $p<0.01$ \\
\hline $\mathrm{V}_{20}$ & $30.7 \%($ range $=9.0-51.6 \%)$ & $53.3 \%($ range $=17.3-75.0 \%)$ & $p<0.01$ \\
\hline $\mathrm{V}_{30}$ & $27.3 \%($ range $=7.5-49.3 \%)$ & $40.9 \%($ range $=10.8-61.2 \%)$ & $p=0.05$ \\
\hline $\mathrm{V}_{40}$ & $22.9 \%($ range $=5.8-46.4 \%)$ & $31.2 \%($ range $=7.7-53.8 \%)$ & $p=0.27$ \\
\hline$v_{50}$ & $19.8 \%($ range $=4.8-42.2 \%)$ & $22.4 \%($ range $=5.9-47.4 \%)$ & $p=0.63$ \\
\hline Mean dose & 17.6 Gy $(\mathrm{RBE})[\mathrm{range}=5.1-30.1 \mathrm{~Gy}(\mathrm{RBE})]$ & 28.8 Gy (range $=11.4-41.0 \mathrm{~Gy})$ & $p<0.05$ \\
\hline
\end{tabular}

$\mathrm{N}=10$. C-ion RT: Carbon ion radiotherapy; $\mathrm{D}_{95}$ : the percentage of the minimum dose that covered $95 \%$ of the target volume; DVH: dose volume histogram; IMRT: intensity-modulated radiotherapy; PTV: planning target volume; RBE: relative biological effectiveness; $\mathrm{V}_{5-50}$ : the percentage of the normal liver volume that received at least 5-50 Gy (RBE) or 5-50 Gy.

Table II. DVH parameters of C-ion RT [60 Gy (RBE)] and simulated IMRT (50 Gy).

\begin{tabular}{|c|c|c|c|}
\hline & Median (range) for C-ion RT at $60 \mathrm{~Gy}(\mathrm{RBE})$ & Median (range) for IMRT at $50 \mathrm{~Gy}$ & $p$-Value \\
\hline PTV D 95 & $58.5 \mathrm{~Gy}(\mathrm{RBE})[$ range=49.1-60.0 Gy (RBE) $]$ & 49.1 Gy (range=46.6-50.0 Gy) & $p<0.01$ \\
\hline \multicolumn{4}{|c|}{ 然 } \\
\hline $\mathrm{V}_{5}$ & $41.0 \%($ range $=12.1-76.2 \%)$ & $82.6 \%($ range $=47.2-99.7 \%)$ & $p<0.01$ \\
\hline$v_{10}^{3}$ & $34.5 \%($ range $=10.6-71.2 \%)$ & $68.1 \%($ range $=29.1-91.9 \%)$ & $p<0.01$ \\
\hline $\mathrm{v}_{20}$ & $30.7 \%($ range $=9.0-51.6 \%)$ & $47.9 \%($ range $=13.7-70.2 \%)$ & $p<0.05$ \\
\hline $\mathrm{v}_{30}$ & $27.3 \%($ range $=7.5-49.3 \%)$ & $35.3 \%($ range $=8.7-56.5 \%)$ & $p=0.32$ \\
\hline $\mathrm{V}_{40}$ & $22.9 \%($ range $=5.8-46.4 \%)$ & $23.9 \%($ range $=6.2-49.0 \%)$ & $p=0.95$ \\
\hline $\mathrm{V}_{50}$ & $19.8 \%($ range $=4.8-42.2 \%)$ & $14.4 \%($ range $=3.6-35.2 \%)$ & $p=0.23$ \\
\hline Mean dose & 17.6 Gy $(\mathrm{RBE})[\mathrm{range}=5.1-30.1 \mathrm{~Gy}(\mathrm{RBE})]$ & 24.0 Gy (range $=9.5-34.2 \mathrm{~Gy})$ & $p=0.15$ \\
\hline
\end{tabular}

N=10. C-ion RT: Carbon ion radiotherapy; D95: the percentage of the minimum dose that covered 95\% of the target volume; DVH: dose volume histogram; IMRT: intensity-modulated radiotherapy; PTV: planning target volume; RBE: relative biological effectiveness; $\mathrm{V}_{5-50}$ : the percentage of the normal liver volume that received at least 5-50 Gy (RBE) or 5-50 Gy.

organ has poor tolerance for radiation. One such study found that an MLD > 33 Gy and $\mathrm{V}_{30}>28 \%$ were risk factors for RILD (17). In the present study, $70-80 \%$ of patients would be difficult to treat with IMRT at 60 Gy owing to the high risk of developing RILD. Moreover, $60-70 \%$ of patients risked developing RILD even with IMRT at $50 \mathrm{~Gy}$. Lowering the prescribed dose would decrease the target $D_{95}$, which would likely worsen the local control rate. In contrast, when administering 60 Gy (RBE) of C-ion RT, $10 \%$ of patients exceeded an MLD of 23 Gy and $40 \%$ exceeded a $V_{30}$ of $28 \%$; the $\mathrm{D}_{95}$ of the dose coverage to the PTV using this method was significantly better than that when using $50 \mathrm{~Gy}$ of IMRT. These data indicate that C-ion RT ought to be preferable to IMRT in terms of delivering a sufficient radiation dose to the $\mathrm{LAHCC}$, while simultaneously avoiding the risk of RILD. Additionally, adopting C-ion RT created the possibility of treating patients with LAHCC who were ineligible for IMRT, thereby expanding the treatment indication for LAHCC.

There was no significant difference in the PTV $\mathrm{D}_{95}$ between the C-ion RT at $60 \mathrm{~Gy}$ (RBE) and IMRT at $60 \mathrm{~Gy}$ groups. However, the lowest PTV $\mathrm{D}_{95}$ among the patients who received C-ion RT was 49.1 Gy (RBE), which was below the lowest PTV $\mathrm{D}_{95}$ observed in the simulated IMRT at $60 \mathrm{~Gy}$. This may be because C-ion RT plans used fewer ports than did IMRT plans. In the present study, C-ion RT treatment plans made by a maximum of three ports to avoid the GI tract maintain the planned constraints while irradiating the PTV to the greatest extent possible, especially when the PTV is surrounded by the GI tract. As such, the dose distribution with $\mathrm{C}$-ion RT could be further improved with the use of additional ports (as is done with IMRT).

Liver functional status is a critical prognostic factor (18, 19). A higher radiation dose-concentration directed at the 

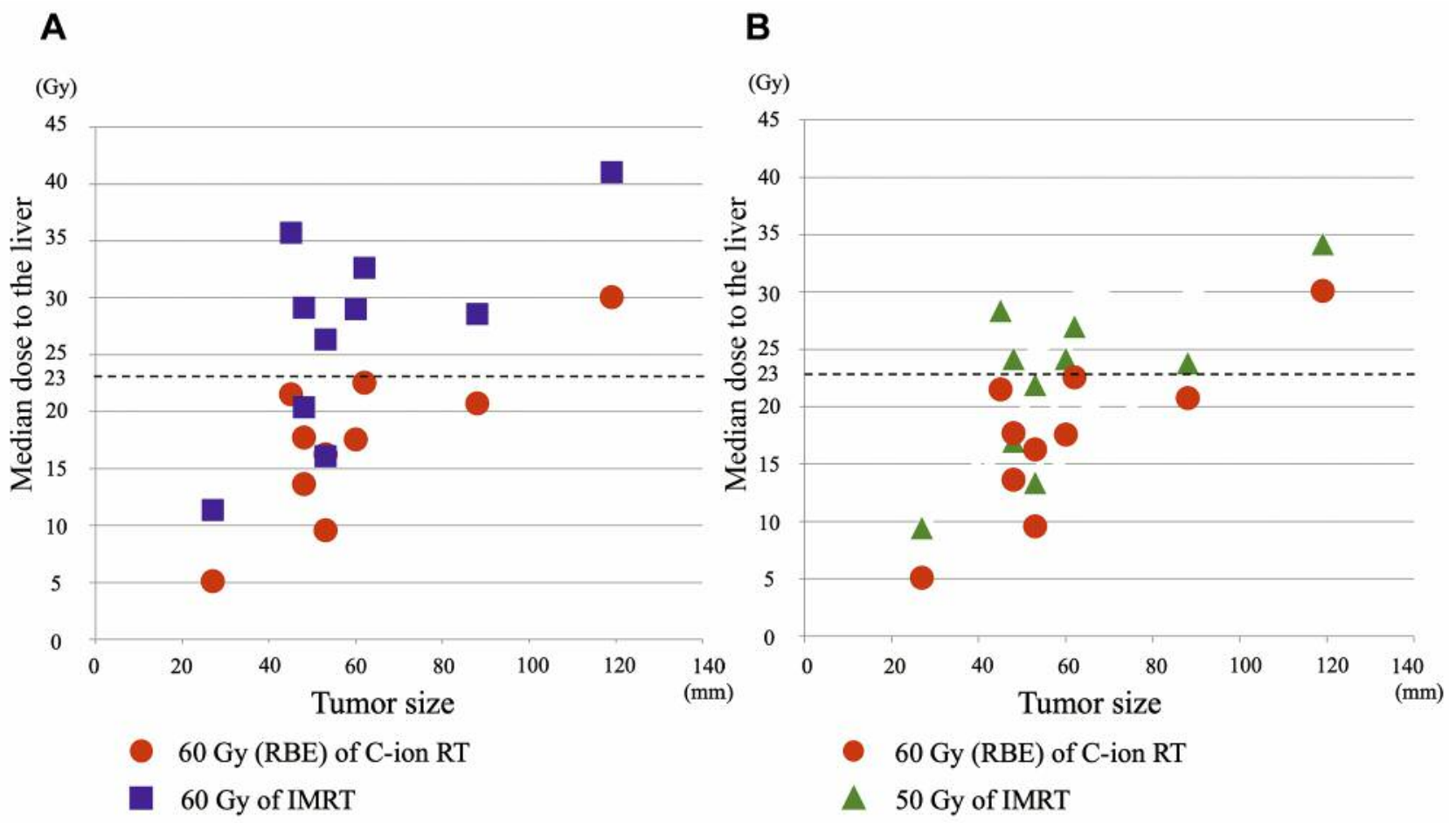

Figure 3. Scatter plots of the mean liver dose (MLD) and tumor size. (A) Comparing 60 Gy [relative biological effectiveness (RBE)] of carbon-ion radiotherapy (C-ion RT) and $60 \mathrm{~Gy}$ of intensity-modulated radiotherapy (IMRT). (B) Comparing $60 \mathrm{~Gy}$ (RBE) of C-ion RT and $50 \mathrm{~Gy}$ of IMRT.

tumor might maintain liver function status and consequently survival. Yoon et al. reported that TACE combined with Xray RT resulted in improved overall and progression-free survival than did sorafenib treatment in patients with LAHCC. Hence, TACE combined with C-ion RT might further improve survival over TACE combined with X-ray $\mathrm{RT}$, particularly as $\mathrm{C}$-ion $\mathrm{RT}$ can help maintain liver function.

There were some limitations in our study. First, we performed a retrospective simulation with a small number of patients. Second, the dose of C-ion RT to the liver [Gy (RBE)] might not be equal to that of IMRT (Gy). Because Gy (RBE) is calculated based on the survival response of human salivary gland cells after $\mathrm{C}$-ion beam irradiation, and not of liver cells, a direct comparison between Gy (RBE) and Gy for the liver can be problematic. Moreover, the dose constraints of C-ion RT and those of IMRT for the liver might be different. However, the dose constraints of $\mathrm{C}$-ion RT for the liver used in our study are considered safe, as previous clinical studies of $\mathrm{C}$-ion $\mathrm{RT}$ used the same dose constraints, and no severe toxicities were observed (7-11).

In conclusion, we demonstrated that $\mathrm{C}$-ion RT for treating LAHCC provides a better dose distribution than IMRT; a clinical-use dose of $60 \mathrm{~Gy}$ (RBE) using C-ion RT showed a superior dose distribution to the PTV and liver than did 50
Gy of IMRT. Additionally, our results suggest that adopting C-ion RT may enable the treatment of patients with LAHCC who cannot be treated with IMRT owing to the high risk of RILD; hence, C-ion RT may expand the treatment indication of LAHCC.

\section{Conflicts of Interest}

The Authors have no conflicts of interest to declare.

\section{Authors' Contributions}

S.S., K.S., M.K., T.N. and T.O. made substantial contributions to the conception and design of the study. S.S., K.S., N.O., T.K., Y.K., and T.O. treated and followed the patients. S.S., K.S, N.O., and T.O. collected the data. S.S., K.S., M.K., and T.O. drafted the manuscript and performed the statistical analysis. N.O., T.K., M.O., Y.K., and T.N. were involved in revising the manuscript critically for important intellectual content. S.S., K.S., and T.O. participated in the acquisition and interpretation of the data. All Authors read and approved the final manuscript.

\section{Acknowledgements}

The Authors wish to thank all the patients involved in this study and our colleagues at the Department of Radiation Oncology, Gunma University Graduate School of Medicine. 


\section{References}

1 Yoon SM, Ryoo BY, Lee SJ, Kim JH, Shin JH, An JH, Lee HC and Lim YS: Efficacy and safety of transarterial chemoembolization plus external beam radiotherapy $v s$. sorafenib in hepatocellular carcinoma with macroscopic vascular invasion: A randomized clinical trial. JAMA Oncol 4(5): 661-669, 2018. PMID: 29543938. DOI: 10.1001/jamaoncol.2017.5847

2 Takeda A, Sanuki N, Tsurugai Y, Iwabuchi S, Matsunaga K, Ebinuma H, Imajo K, Aoki Y, Saito H and Kunieda E: Phase 2 study of stereotactic body radiotherapy and optional transarterial chemoembolization for solitary hepatocellular carcinoma not amenable to resection and radiofrequency ablation. Cancer 122(13): 2041-2049, 2016. PMID: 27062278. DOI: 10.1002/cncr.30008

3 Wang WH, Wang Z, Wu JX, Zhang T, Rong WQ, Wang LM, Jin J, Wang SL, Song YW, Liu YP, Ren H, Fang H, Wang WQ, Liu $\mathrm{XF}$, Yu ZH and Li YX: Survival benefit with IMRT following narrow-margin hepatectomy in patients with hepatocellular carcinoma close to major vessels. Liver Int 35(12): 2603-2610, 2015. PMID: 25939444. DOI: 10.1111/liv.12857

4 Bae SH, Jang WI and Park HC: Intensity-modulated radiotherapy for hepatocellular carcinoma: dosimetric and clinical results. Oncotarget 8(35): 59965-59976, 2017. PMID: 28938697. DOI: 10.18632/oncotarget.19219

5 Abe T, Saitoh J, Kobayashi D, Shibuya K, Koyama Y, Shimada H, Shirai K, Ohno T and Nakano T: Dosimetric comparison of carbon ion radiotherapy and stereotactic body radiotherapy with photon beams for the treatment of hepatocellular carcinoma. Radiat Oncol 10: 187, 2015. PMID: 26377092. DOI: 10.1186/ s13014-015-0491-8

6 Toramatsu C, Katoh N, Shimizu S, Nihongi H, Matsuura T, Takao S, Miyamoto N, Suzuki R, Sutherland K, Kinoshita R, Onimaru R, Ishikawa M, Umegaki $\mathrm{K}$ and Shirato $\mathrm{H}$ : What is the appropriate size criterion for proton radiotherapy for hepatocellular carcinoma? A dosimetric comparison of spot-scanning proton therapy versus intensity-modulated radiation therapy. Radiat Oncol 8: 48, 2013. PMID: 23497543. DOI: 10.1186/1748-717X-8-48

7 Shiba S, Abe T, Shibuya K, Katoh H, Koyama Y, Shimada H, Kakizaki S, Shirabe K, Kuwano H, Ohno T and Nakano T: Carbon ion radiotherapy for 80 years or older patients with hepatocellular carcinoma. BMC Cancer 17(1): 721, 2017. PMID: 29115938. DOI: 10.1186/s12885-017-3724-4

8 Shibuya K, Ohno T, Katoh H, Okamoto M, Shiba S, Koyama Y, Kakizaki S, Shirabe K and Nakano T: A feasibility study of highdose hypofractionated carbon ion radiation therapy using four fractions for localized hepatocellular carcinoma measuring $3 \mathrm{~cm}$ or larger. Radiother Oncol 132: 230-235, 2019. PMID: 30366726. DOI: 10.1016/j.radonc.2018.10.009

9 Shibuya K, Ohno T, Terashima K, Toyama S, Yasuda S, Tsuji H, Okimoto T, Shioyama Y, Nemoto K, Kamada T and Nakano T; Japan Carbon Ion Radiotherapy Study Group: Short-course carbon-ion radiotherapy for hepatocellular carcinoma: A multiinstitutional retrospective study. Liver Int 38(12): 2239-2247, 2018. PMID: 30240527. DOI: 10.1111/liv.13969

10 Shiba S, Shibuya K, Katoh H, Kaminuma T, Miyazaki M, Kakizaki S, Shirabe K, Ohno T and Nakano T: A comparison of carbon ion radiotherapy and transarterial chemoembolization treatment outcomes for single hepatocellular carcinoma: a propensity score matching study: Radiat Oncol 14(1): 137, 2019. PMID: 31375120. DOI: 10.1186/s13014-019-1347-4
11 Kasuya G, Kato H, Yasuda S, Tsuji H, Yamada S, Haruyama Y, Kobashi G, Ebner DK, Okada NN, Makishima H, Miyazaki M, Kamada $\mathrm{T}$ and Tsujii $\mathrm{H}$; Liver Cancer Working Group: Progressive hypofractionated carbon-ion radiotherapy for hepatocellular carcinoma: Combined analyses of 2 prospective trials. Cancer 123(20): 3955-3965, 2017. PMID: 28662297. DOI: $10.1002 /$ cncr.30816

12 Abe S, Kubota Y, Shibuya K, Koyama Y, Abe T, Ohno T and Nakano T: Fiducial marker matching versus vertebral body matching: Dosimetric impact of patient positioning in carbon ion radiotherapy for primary hepatic cancer. Phys Med 33: 114-120, 2017. PMID: 28057427. DOI: 10.1016/j.ejmp.2016.12.018

13 Kubota Y, Katoh H, Shibuya K, Shiba S, Abe S, Sakai M, Yuasa D, Tsuda K, Ohno T and Nakano T: Comparison between bone matching and marker matching for evaluation of intra- and interfractional changes in accumulated dose of carbon ion radiotherapy for hepatocellular carcinoma. Radiother Oncol 137: 77-82, 2019. PMID: 31078014. DOI: 10.1016/j.radonc.2019.04.026

14 Tsujii H, Kamada T, Shirai T, Noda K, Tsuji H and Karasawa K: Carbon-ion radiotherapy: Principles, practices, and treatment planning. Tokyo: Springer; 2014.

15 Kanematsu N: Dose calculation algorithm of fast fineheterogeneity correction for heavy charged particle radiotherapy. Phys Med 27(2): 97-102, 2011. PMID: 20579913. DOI: 10.1016/j.ejmp.2010.05.001

16 Inaniwa T, Kanematsu N, Matsufuji N, Kanai T, Shirai T, Noda K, Tsuji H, Kamada T and Tsujii H: Reformulation of a clinicaldose system for carbon-ion radiotherapy treatment planning at the National Institute of Radiological Sciences, Japan. Phys Med Biol 60(8): 3271-3286, 2015. PMID: 25826534. DOI: 10.1088/ 0031-9155/60/8/3271

17 Liang SX, Zhu XD, Xu ZY, Zhu J, Zhao JD, Lu HJ, Yang YL, Chen L, Wang AY, Fu XL and Jiang GL: Radiation-induced liver disease in three-dimensional conformal radiation therapy for primary liver carcinoma: The risk factors and hepatic radiation tolerance. Int J Radiat Oncol Biol Phys 65(2): 426-434, 2006. PMID: 16690430. DOI: 10.1016/j.ijrobp.2005.12.031

18 Llovet JM, Real MI, Montaña X, Planas R, Coll S, Aponte J, Ayuso C, Sala M, Muchart J, Solà R, Rodés J and Bruix J; Barcelona Liver Cancer Group: Arterial embolisation or chemoembolisation versus symptomatic treatment in patients with unresectable hepatocellular carcinoma: a randomised controlled trial. Lancet 359(9319): 1734-1739, 2002. PMID: 12049862. DOI: 10.1016/S0140-6736(02)08649-X

19 Mathurin P, Rixe O, Carbonell N, Bernard B, Cluzel P, Bellin MF, Khayat D, Opolon P and Poynard T: Review article: Overview of medical treatments in unresectable hepatocellular carcinoma - an impossible meta-analysis? Aliment Pharmacol Ther 12(2): 111-126, 1998. PMID: 9692685. DOI: 10.1046/j.1365-2036.1998.00286.x 